\title{
ANNUAL PROGRESS REPORT FY92
}

\section{Grant: DE-FG02-88ER45367}

A Free Energy Simulation Method Based Study of Interfacial Segregation

Submitted by: D. J. Srolovitz, University of Michigan 3/18/93

Within the past year we have employed our free energy minimization method to investigate the order-disorder at, segregation to, and thermodynamic properties of grain boundaries and surfaces, and to determine the thermodynamic properties of metastable phases in binary alloys. We have also begun exploring the applicability of our free energy minimization method to materials possessing structure that are more open than the fcc metals that have been our focus to date.

In the free energy minimization method one minimizes an approximate free energy functional with respect to all atom positions and concentration profiles. In this approximation each atom is assumed to vibrate independent of the other atoms and its vibration is characterized by three frequencies which may be determined by diagonalizing a $(3 \times 3)$ local dynamical matrix. In an alloy system the configurational entropy contribution to the free energy is approximated by Bragg-Williams method.

Segregation to and the thermodynamics of twist grain boundaries in $\mathrm{Cu}-\mathrm{Ni}$ with misorientation angle ranging from $8^{\circ}$ to $44^{\circ}$ were investigated over a wide range of temperature. It was demonstrated that the structural unit model (in which the structure of a grain boundary in an elemental system is decomposed to the structures of the two delimiting, special grain boundaries) is equally applicable to the structure of the grain boundaries in binary alloys. It was also established that there is a strong relationship between the magnitude of the local hydrostatic pressure and the degree of segregation. The degree of segregation to sites with high hydrostatic pressure is stronger than to sites with low hydrostatic pressure. An overall effect of segregation is to make the hydrostatic pressure field in the grain boundary region more uniform than it is in the absence of segregation.

Segregation to and order-disorder phase transitions at grain boundaries in the ordered intermetallic $\mathrm{Ni}_{3-\mathrm{x}} \mathrm{Al}_{1+\mathrm{x}}$ were also investigated. A first order grain boundary phase transition from the high temperature $\mathrm{L}_{2}$ ordered phase ( $\mathrm{Ni}_{3} \mathrm{Al}$ structure) to the low temperature $\mathrm{L} 1_{0}$ ordered phase (NiAl structure) was observed on cooling alloys rich in $\mathrm{Al}(x>0)$ from high temperature. It was also demonstrated that the effect of $\mathrm{Ni}$ or Al segregation to grain boundaries on the ideal cohesive energy is very small. In light of these results, we speculated that the formation of the brittle $A B$ (NiAl) phase at the grain boundaries in ordered intermetallic $A_{3} B$ ( $\mathrm{Ni}_{3} \mathrm{Al}$ ) alloys with an $\mathrm{LI}_{2}$ structure at low temperature is responsible for the intergranular fracture behavior of these alloys. The effect of alloying these materials with boron or other impurities may be to remove this brittle $A B$ phase from the grain boundaries.

Order-disorder transitions at and segregation to the (001), (011), and (111) surfaces in $\mathrm{Pd}-\mathrm{Cu}, \mathrm{Pd}-\mathrm{Ag}$, and $\mathrm{Pd}-\mathrm{Au}$ alloys were investigated. It was observed that

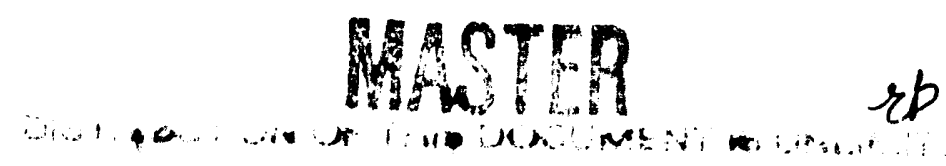


- the (001) surface in Cu-rich alloys undergoes a surface phase transition from a disordered to an ordered surface phase upon cooling from high temperature. This transition is similar to the (001) surface transition in Ni-rich $\mathrm{Pt}-\mathrm{Ni}$ alloys, which we have previously reported. The surface transition temperature is well above the bulk order-disorder transition temperature. However, the (001) surfaces with slight miscuts (i.e. vicinal surfaces) underwent surface order-disordered phase transition at a lower temperature than that of the flat (001) surface. At temperatures above the surface transition temperature for these surfaces we observed some local compositional ordering initiated at the step site and extended though with less pronounced on the (001) ledge of the surface.

Thermodynamic properties of and concentration profile in small spheres of binary alloys were determined. In these studies, where clusters of up 70,000 atoms were considered, we calculated the "average" surface free energy and surfaces stress tensor for a series of cluster sizes at different temperatures. This calculation provides good measures for the ratios of average surface energy and the energy of the surfaces with high symmetry. From stereographic projections of the surface energy density and the excess segregation density, the surface with the highest and lowest free energy and the surfaces with highest and lowest degree of segregation were determined, respectively. The segregation and ordering appear to be correlated.

The free energy minimization method was also used to calculate the heat of formation and the lattice parameter of $\mathrm{Ag}-\mathrm{Cu}$ metastable phases. The results were in good agreement with the experimental measurements made by our collaborators. This method provides an alternative approach to the more traditional thermodynamic methods such as CalPhad which significantly underestimates the heat of formation for $\mathrm{Ag}-\mathrm{Cu}$ metastable phases.

Our preliminary studies on the thermodynamics properties of perfect $\mathrm{Si}$ solid shows promising results. The results of our free energy minimization for the free energy and entropy of $\mathrm{Si}$ are in excellent agreement with experimental data and quasiharmonic calculations. 

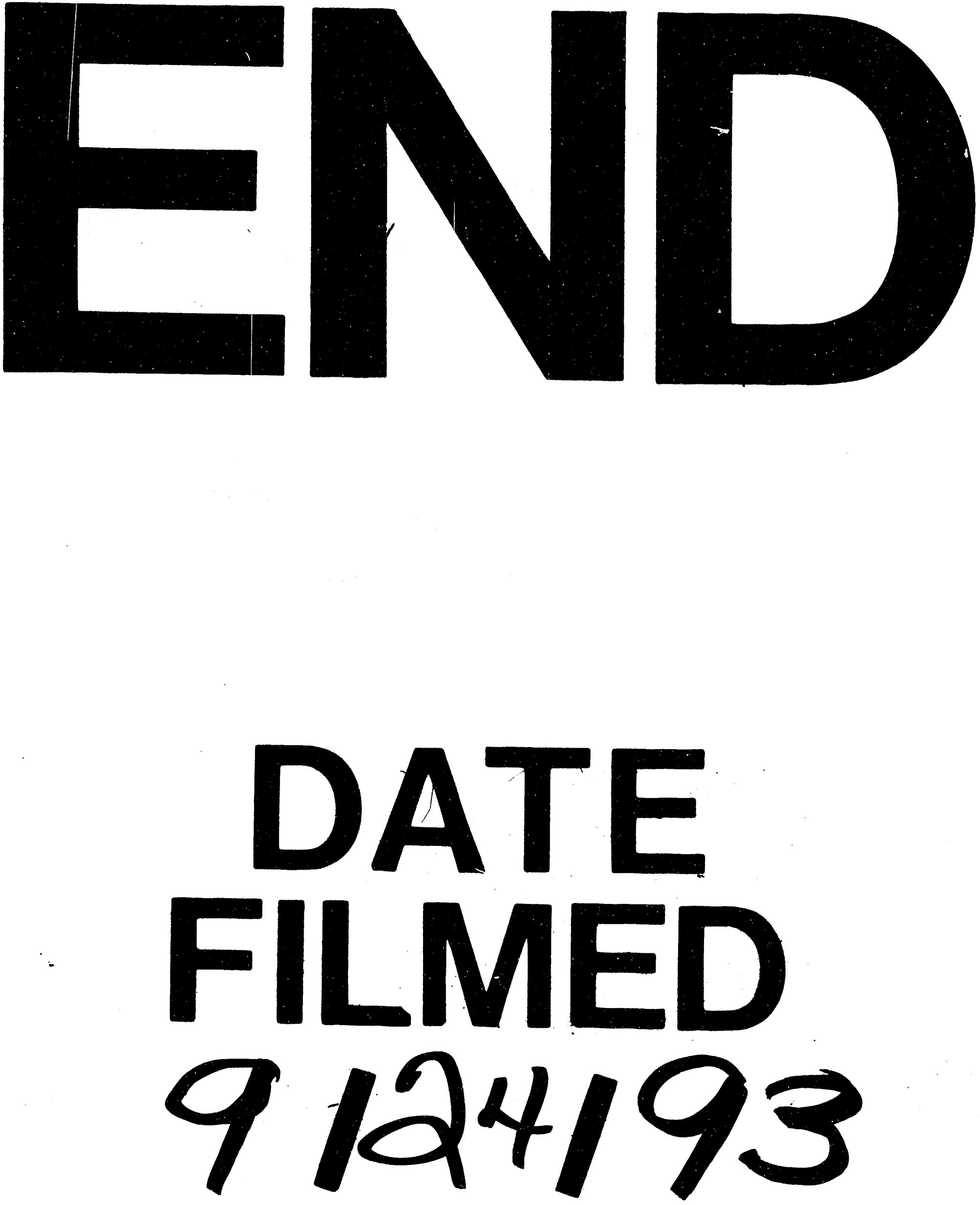
